\title{
Disability in patients consulting for anxiety or mood disorders in primary care: response to antidepressant treatment
}

This article was published in the following Dove Press journal:

Neuropsychiatric Disease and Treatment

12 December 2012

Number of times this article has been viewed

\author{
Alain Gérard' \\ François Liard ${ }^{2}$ \\ Anne Crochard ${ }^{3}$ \\ Sylvia Goni ${ }^{3}$ \\ Bruno Millet ${ }^{4}$ \\ 'Rue des Marronniers' Psychiatry \\ Clinic, Paris; ${ }^{2}$ General Practice, Saint \\ Epain, ${ }^{3}$ Medical Affairs Department, \\ Laboratoire Lundbeck SAS, Issy-Les- \\ Moulineaux, ${ }^{4}$ Psychiatry Department, \\ Guillaume Regner University Hospital, \\ University of Rennes I, Rennes, \\ France
}

Background: The primary objective of this prospective observational study was to evaluate changes in self-reported disability in patients with anxiety or mood disorders 3 months after initiating antidepressant treatment.

Methods: This study included 8396 patients consulting 2433 general practitioners in France for a major mood episode, generalized anxiety disorder, social anxiety disorder, panic disorder, or obsessive-compulsive disorder. Treatment was initiated with the antidepressant that the physician considered appropriate. Patients were evaluated with the Sheehan Disability Scale (SDS), Hospital Anxiety and Depression Scale, and Clinical Global Impression-Severity (CGI-S) at baseline and after 6 and 12 weeks.

Results: At 12 weeks, 6617 patients (78.8\%) were evaluable. At inclusion, the mean SDS subscores were $6.5 \pm 2.2$ on the work/school activities dimension, $6.8 \pm 1.9$ on the social activities dimension, and $6.5 \pm 2.0$ on the family life dimension. At the 12-week follow-up visit, the mean change in score on these three dimensions was $-3.9 \pm 2.6,-4.2 \pm 2.5$, and $-4.0 \pm 2.5$, respectively. At the 12-week follow-up visit, $90.0 \%$ of patients were responders (defined as patients whose SDS dimension scores decreased by at least one point) on the work/school SDS subscores; $92.8 \%$ were responders on the social life SDS subscores, and $91.1 \%$ were responders on family life/ home responsibilities SDS subscores. Functional remission (defined as an SDS subscore of 0 at study end) rates were $18.0 \%$ for the work/school dimension, $16.8 \%$ for the social activities dimension, and $19.5 \%$ for the family life dimension. Using a cutoff of $\leq 2$, remission rates were $56.8 \%, 55.0 \%$, and $58.0 \%$, respectively. Improvements in self-rated disability were correlated with improvements in symptoms measured with clinician-rated CGI-S.

Conclusion: Patients consulting for anxiety or mood disorders report significant disability, which can be effectively reduced by antidepressant treatment.

Keywords: depression, anxiety disorder, antidepressant, disability

\section{Introduction}

Anxiety and mood disorders are the most frequent mental disorders identified in most epidemiological surveys of mental health conducted in the general population. ${ }^{1}$ The World Health Organization (WHO) World Mental Health Survey (WMH) initiative, which has collected data on the epidemiology and burden of mental disorders in 28 countries using a standardized methodology, has reported worldwide 12-month prevalence rates of around $6 \%$ for mood disorders and around $11 \%$ for anxiety disorders. ${ }^{1}$ In France, a general population survey carried out within the framework of the WMH survey identified a 12-month prevalence rate of mood, anxiety, and alcohol use disorders of $18.9 \%$, of which the most frequent individual disorder was
Correspondence: Bruno Millet Centre Hospitalier Guillaume Regnier, 108 Av du General Leclerc, 35703 Rennes Cedex 7, France $\mathrm{Tel}+33299333937$

$\mathrm{Fax}+33299333972$

Email bruno.millet@univ-rennes I.fr 
major depressive disorder, with a 12-month prevalence rate of $7.4 \% .^{2}$ The lifetime prevalence rates for mood and anxiety disorders in this study were $24.1 \%$ and $22.4 \%$, respectively. These figures place France among the countries with a relatively high prevalence of these disorders. In addition, comorbidity between mood and anxiety disorders is high. For example, in the European Study of the Epidemiology of Mental Disorders in six European countries, 53\% of individuals with major depressive disorder, $69 \%$ of those with generalized anxiety disorder, and $64 \%$ of those with panic disorder presented with another mood or anxiety disorder. ${ }^{3}$ Similar findings have been reported by the $\mathrm{US}^{4}$ and Australia. ${ }^{5}$ The extensive comorbidity between mood and anxiety disorders has inspired consideration of a more dimensional approach to the classification of these disorders, and it is anticipated that this will be one of the innovations in the revised Diagnostic and Statistical Manual of Mental Disorders Fifth Edition (DSM-V) diagnostic criteria currently in preparation. ${ }^{6}$

In addition to being common, mood and anxiety disorders are also disabling. Indeed, the WHO Global Burden of Disease report identified unipolar mood disorders as the third most disabling disease after lower respiratory infection and diarrheal disease, in terms of years of healthy life due to poor health or disability. ${ }^{7}$ In the WMH surveys, disability has been assessed using a self-reported rating scale, ie, the Sheehan Disability Scale (SDS). ${ }^{8}$ The proportion of individuals reporting severe disability ranged from $48.4 \%$ for panic disorder to $65.8 \%$ for major depressive disorder, which was higher than for all the physical disorders evaluated, including chronic pain $(40.9 \%)$, arthritis (23.3\%), and cancer $(16.6 \%) .{ }^{8}$ This disability is associated with reduced productivity ${ }^{9,10}$ and impaired quality of life, ${ }^{11,12}$ emphasizing the significant social burden of mood and anxiety disorders. The relevance of disability and its evaluation is expected to be another theme of the forthcoming DSM-V Text Revision classification of psychiatric disorders. ${ }^{6}$

Several randomized clinical trials have used the SDS as an outcome measure to evaluate the impact of antidepressant treatment on self-reported disability in patients with major depressive disorder or various anxiety disorders, as reviewed by Sheehan and Sheehan in 2008. ${ }^{13}$ These have generally reported reductions in mean SDS disability scores within 12 weeks of initiation of antidepressant treatment. Changes in mean scores and the distribution of scores between different disability levels in placebo-controlled trials have consistently favored antidepressant treatment over placebo. ${ }^{13}$

Nonetheless, information on the impact of antidepressant treatment on disability in everyday treatment settings is sparse, and few naturalistic treatment studies of antidepressants have measured disability as an outcome. For this reason, we undertook a large naturalistic study of disability in patients with mood or anxiety disorders who initiate treatment with an antidepressant in primary care in France, known as DéPasS (Depression, Anxiety and Disability: patient follow-up in general practice). The primary objective of the study was to evaluate the change in self-reported disability in patients with anxiety or mood disorders 3 months after initiating antidepressant treatment.

\section{Materials and methods}

This was a prospective, observational study conducted in a naturalistic primary care treatment setting in France between March 2009 and February 2010.

\section{Investigators}

The study investigators were randomly selected from a representative list of 30,000 general practitioners, who, in turn, were identified from an exhaustive list of all general practitioners licensed by the national medical association in France and in active practice (Cegedim OneKey registry). All physicians were contacted by mail and invited to participate in the study. Physicians wishing to participate were provided with the study documentation by mail. No attempt was made to follow up physicians who failed to reply.

\section{Patients}

It was anticipated that each participating general practitioner would include around four eligible patients who spontaneously consulted for an anxiety or mood disorder during the 3 months following receipt of the study documentation. Adult patients ( $\geq 18$ years old) presenting with any of the following diagnoses were eligible for the study: major depressive disorder, generalized anxiety disorder, social anxiety disorder, panic disorder, or obsessive-compulsive disorder. These diagnoses were made by the physician based on their experience and clinical judgment. The DSM-IV Text Revision diagnostic criteria (American Psychiatric Association, 2000) for the diagnoses of interest were provided to all participating general practitioners, together with the study protocol. Only patients for whom the general practitioner had decided, either prior to or during the index consultation, to prescribe an antidepressant treatment were included. Patients were required to possess the necessary cognitive and linguistic skills to be able to complete the questionnaires, and to provide their verbal consent to participate in the study. Patients who were currently participating in a clinical research study were excluded from the study. 


\section{Target sample size}

The number of patients to be included was determined by a priori power calculations. In order to estimate response rates on the SDS at 3 months with a $95 \%$ confidence interval of $4.5 \%$ for a frequency of $50 \%$ in each diagnostic subgroup and a two-sided $\alpha$ risk of 0.05 , it would be necessary to evaluate at least 474 patients with each of the diagnoses of interest. Assuming a dropout rate of $20 \%$ over the 12 -week follow-up period, it would be necessary to include 593 patients in order to have 474 patients evaluable by diagnosis at study end. Based on the published relative prevalence of anxiety and mood disorders in France, ${ }^{2}$ panic disorder would be expected to be the least represented of these disorders in patients consulting a general practitioner, accounting for $9 \%$ of patients. Thus, in order to ensure inclusion of around 593 patients with panic disorder, it would be necessary to include 6588 patients with anxiety or mood disorders.

Given that each participating investigator was expected to include a minimum of two patients with an anxiety or mood disorder within 3 months, 3000 participating general practitioners would be required. Overall, 30,000 general practitioners were contacted, assuming a positive response rate of $<15 \%$ and that three-quarters of physicians who accepted to participate would actually include patients.

\section{Study procedures}

Data were collected at the index consultation (inclusion visit) and at two follow-up consultations about 6 and 12 weeks after the index consultation. A window of \pm 2 weeks was considered acceptable for the 6-week visit and a window of \pm 3 weeks for the 12 -week visit. These visits were programmed as part of the routine follow-up of patients, and no additional protocol-specified study visit was imposed. The choice of antidepressant and treatment modalities, such as dose, was made by the investigator. If the treatment was discontinued before the end of the 12-week follow-up period, this was to be documented, but the patient was still followed and evaluated.

\section{Data collection}

Data were collected either on a paper questionnaire or through a web-based interface, according to the physician's preference. At the inclusion visit, the investigator collected data on sociodemographics, medical history, psychiatric diagnosis, and prescription of antidepressants and other psychotropic drugs. The investigator completed the Clinical Global Impression-Severity (CGI-S) rating scale ${ }^{14}$ at each study visit and the Clinical Global Impression-Improvement
(CGI-I) rating scale at the 6-week and 12-week study visits. In addition, patients completed two questionnaires, the $\mathrm{SDS}^{15}$ and the Hospital Anxiety and Depression Scale (HADS), ${ }^{16}$ at each study visit.

\section{Outcome measures}

The primary outcome measure was the mean change in SDS subscale scores between inclusion and 12 weeks. This patient-reported outcome measure assesses functional impairment in three domains, namely work/school activities, social activities, and family life. For each dimension, the patient rates the extent of impairment over the previous week due to their symptoms on a 10-point visual analog scale, where 0 represents no impairment and 10 represents extreme impairment. The SDS also collects information on the number of days off school/work (or unable to carry out activities of daily living) and the number of days of impaired productivity during the previous week. The proportion of patients scoring $\geq 7$ on each dimension score, corresponding to marked impairment, and the proportion of responders, defined as patients whose SDS dimension scores decreased by at least one point, ${ }^{13}$ were also determined. Functional remission was defined using two cutoffs at study end, namely an SDS subscore of 0 and a subscore of $\leq 2 .{ }^{13}$ It should be noted that, for this analysis, patients with missing data were excluded from the denominator. This was required in order to have comparable values across the three dimension scales because patients not in employment for reasons other than their anxiety or mood disorder are de facto counted as missing for the work/school dimension.

Secondary outcome measures were the change in mean score on the HADS anxiety and depression dimensions between inclusion and 12 weeks, mean change in score on the CGI-S between inclusion and 12 weeks, and the CGI-I score at 12 weeks. The HADS is a patient-reported outcome measure assessing the severity of anxiety and mood symptoms. It has 14 items, seven relating to anxiety and seven to depression, each of which is scored on a four-point Likert scale. Possible scores for each dimension range from 0 to 28, with a higher score reflecting greater severity. A score $\geq 8$ on either dimension corresponds to clinically relevant symptoms. The CGI-S and CGI-I scales are scored on seven-point Likert scales rated by the investigator. Response was defined as a score of 1 or 2 on the CGI-I at 12 weeks, and remission as a score or 1 or 2 on the CGI-S at 12 weeks. In addition, outcome at 6 weeks was assessed using all three outcome measures. 


\section{Statistical analysis}

The primary analysis was an observed cases analysis of the per-protocol set performed for all patients included into the study without protocol violations and for whom SDS outcome data was available for at least one post-baseline study visit. Missing data were not imputed. For data on baseline characteristics and the primary outcome measure (SDS subscales), subgroup analyses were performed by diagnostic group. The analysis is principally descriptive. Statistical comparison of rating scale scores between inclusion and the follow-up visits was performed using the paired Student's $t$-test; categorical variables were compared using the Chi-square test. All testing was two-tailed, and a probability threshold of 0.05 was taken as statistically significant. All data analysis was performed using $\mathrm{SAS}^{\circledR}$ software version 9.1 (SAS Institute, Cary, NC).

\section{Ethics}

The study was performed according to international and French regulatory guidelines and current codes of good epidemiological practice. The aims and procedures of the study were explained and each patient provided their informed consent. Because patient care was not altered by inclusion in the study, ethics committee approval was not required. Data handling for the study was authorized by the CCTIRS (Comité Consultatif sur le Traitement de l'Information en matière de Recherche dans le domaine de la Santé: French Advisory Committee on Information Processing in Health Research) and the CNIL (Commission Nationale d'Informatique et de Libertés: French National data protection authority).

\section{Results \\ Patients}

Overall, 2433 general practitioners participated in the study and included 8396 patients. Of these patients, 6977 (83.1\%) attended a follow-up visit within the prespecified tolerance window at 6 weeks, and $6617(78.8 \%$ ) at 12 weeks (Figure 1). There was no marked difference in the rate of retention at 12 weeks between patients with different diagnoses, ranging from $75.8 \%$ for patients with social anxiety disorder to $79.9 \%$ in patients with obsessive-compulsive disorder. The most frequent diagnosis was major depressive episode $(n=6270$, $74.7 \%$ ) and the least frequent obsessive-compulsive disorder ( $\mathrm{n}=373,4.4 \%$, Table 1). However, multiple diagnoses were reported for many patients $(\mathrm{n}=2280,27.2 \%)$; most frequently major depressive episode was comorbid with an anxiety disor$\operatorname{der}(\mathrm{n}=1871,82.1 \%$ of patients with comorbid disorders), and, for all diagnoses except major depressive episode, comorbid diagnoses were more frequent than isolated disorders.

The sociodemographic features of the patients are presented in Table 2. Their mean age was 48.7 years, and $67.7 \%$ were women. Around half were married (58.3\%) and about one-third (33.2\%) had a university education. Overall, 66.1\% were in employment (employee, self-employed, or doing temporary work) at the time of the study. The sociodemographic

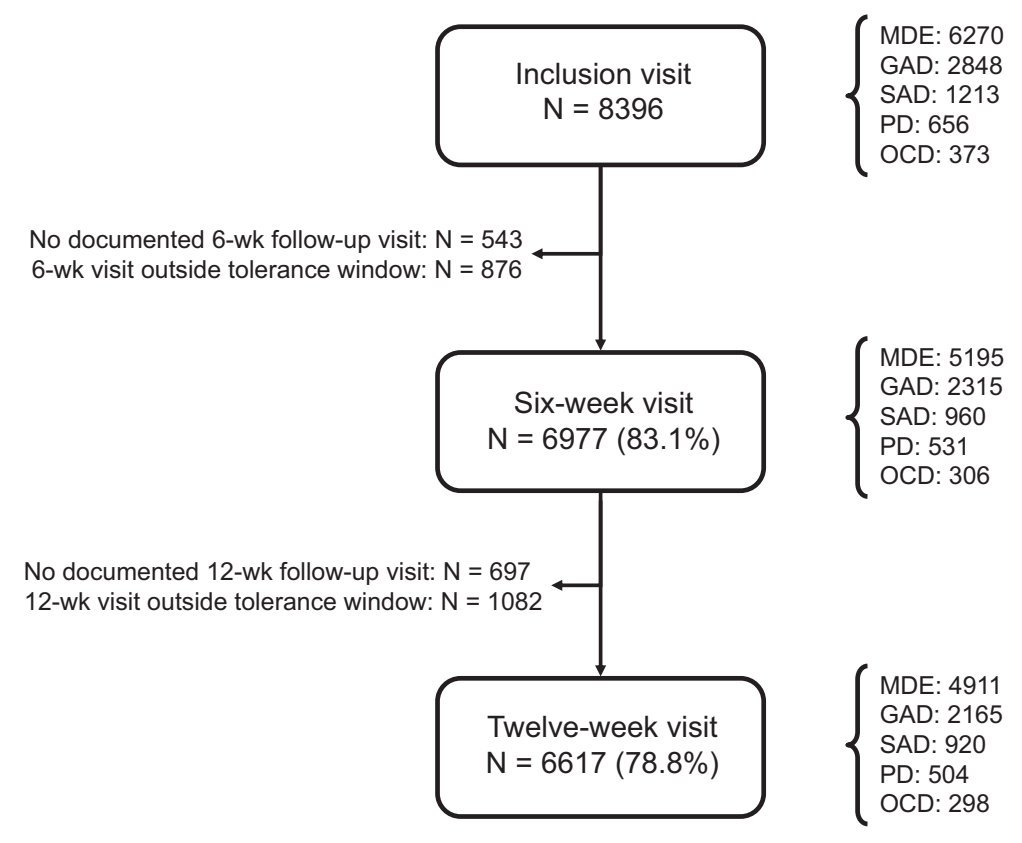

Figure I Patient flow through the study.

Note: The tolerance windows for the 6-week and 12-week follow-up visits were 2 and 3 weeks, respectively.

Abbreviations: MDE, major depressive episode; GAD, generalized anxiety disorder; SAD, social anxiety disorder; PD, panic disorder; OCD, obsessive-compulsive disorder. 
Table I Patient diagnosis

\begin{tabular}{ll}
\hline Diagnosis & N (\%) \\
\hline All MDE & $6270(74.7 \%)$ \\
All GAD & $2848(33.9 \%)$ \\
All SAD & $1213(14.5 \%)$ \\
All PD & $656(7.8 \%)$ \\
All OCD & $373(4.4 \%)$ \\
Only one diagnosis & $6616(72.8 \%)$ \\
MDE alone & $4399(52.4 \%)$ \\
GAD alone & $1137(13.5 \%)$ \\
SAD alone & $268(3.2 \%)$ \\
PD alone & $172(2.0 \%)$ \\
OCD alone & $140(1.7 \%)$ \\
Two diagnoses & $1734(20.7 \%)$ \\
Three or more diagnoses & $546(6.5 \%)$ \\
\hline
\end{tabular}

Note: The total number of patients evaluated was 8396.

Abbreviations: MDE, major depressive episode; GAD, generalised anxiety disorder; SAD, social anxiety disorder; PD, panic disorder; $O C D$, obsessivecompulsive disorder.

features were generally similar between the different diagnostic groups, although patients with obsessive-compulsive disorder were more frequently male and single (Table 2).

\section{Treatments}

The treatments most commonly prescribed at inclusion were selective serotonin reuptake inhibitors, which accounted for $84.7 \%$ of all antidepressants prescribed, the remainder being principally serotonin and noradrenaline reuptake inhibitors (venlafaxine, duloxetine, or milnacipran; $13.0 \%$ of prescriptions). More than one antidepressant was prescribed to 118 patients (1.4\%). For the majority of patients for whom information was available (6562 of 8351, 78.6\%), this was their first antidepressant prescription for the current episode. Antidepressant treatment had been discontinued at or before the 6 -week study visit for $3.3 \%$ of patients $(n=211)$. By the end of the study (12-week follow-up visit), this proportion had risen to $10.1 \%(n=609)$.

In addition to antidepressants, $62.4 \%$ of patients $(\mathrm{n}=5236)$ were coprescribed one or more additional psychotropic drugs, most frequently anxiolytics (4635 patients, 55.2\%) and hypnotics (2286 patients, 27.2\%). Antipsychotics were prescribed to 225 patients $(2.7 \%)$.

\section{Effectiveness}

The primary outcome measure was the change from inclusion in the mean score on the three SDS dimensions. At inclusion, the mean SDS scores were 6.5 on the work/school activities dimension, 6.8 on the social activities dimension, and 6.5 on the family life dimension. At the 12-week follow-up visit, the mean change in score on these three dimensions

Table 2 Sociodemographic features of the study sample

\begin{tabular}{|c|c|c|c|c|c|c|}
\hline & $\begin{array}{l}\text { Total } \\
(N=8396)\end{array}$ & $\begin{array}{l}\text { MDE } \\
(N=6270)\end{array}$ & $\begin{array}{l}\text { GAD } \\
(N=2848)\end{array}$ & $\begin{array}{l}\text { SAD } \\
(N=12 \mid 3)\end{array}$ & $\begin{array}{l}\text { PD } \\
(N=656)\end{array}$ & $\begin{array}{l}\text { OCD } \\
(N=373)\end{array}$ \\
\hline Age & $N=8335$ & $N=6219$ & $N=2820$ & $N=1200$ & $N=648$ & $N=37 I$ \\
\hline Mean \pm SD & $48.7 \pm 14.3$ & $49.4 \pm 14.1$ & $48.6 \pm 14.5$ & $46.6 \pm 13.3$ & $46.4 \pm 14.7$ & $45.7 \pm 14.6$ \\
\hline Median [range] & $48[18 ; 97]$ & $49[18 ; 97]$ & $48[19 ; 93]$ & $47[19 ; 92]$ & $46[18 ; 89]$ & $44[18 ; 95]$ \\
\hline Gender & $N=8335$ & $N=6224$ & $N=2827$ & $N=1202$ & $N=650$ & $N=372$ \\
\hline Male & 2689 (32.3\%) & I94| (31.2\%) & $892(31.6 \%)$ & 419 (34.9\%) & 207 (31.8\%) & 136 (36.6\%) \\
\hline Female & 5646 (67.7\%) & 4283 (68.8\%) & 1935 (68.4\%) & $783(65.1 \%)$ & $443(68.2 \%)$ & 236 (63.4\%) \\
\hline Marital status & $N=8363$ & $N=6242$ & $N=2843$ & $N=1207$ & $N=654$ & $N=372$ \\
\hline Married & 4873 (58.3\%) & 3630 (58.2\%) & 1680 (59.1\%) & $678(56.2 \%)$ & $376(57.5 \%)$ & 192 (51.6\%) \\
\hline Single & II 86 (I4.2\%) & 763 (I2.2\%) & $429(15.1 \%)$ & $218(18.1 \%)$ & II (I8.0\%) & 96 (25.8\%) \\
\hline Separated & I67| (20.0\%) & | 322 (2|.2\%) & $520(18.3 \%)$ & 249 (20.6\%) & 127 (19.4\%) & $66(17.7 \%)$ \\
\hline Widowed & 633 (7.6\%) & 527 (8.4\%) & 214 (7.5\%) & $62(5.1 \%)$ & $33(5.0 \%)$ & 18 (4.8\%) \\
\hline Educational level & $N=835 I$ & $N=6230$ & $N=2837$ & $N=1207$ & $N=653$ & $N=373$ \\
\hline Primary & 904 (10.8\%) & 683 (II.0\%) & 345 (12.2\%) & 145 (I2.0\%) & 64 (9.8\%) & 52 (I3.9\%) \\
\hline Secondary & $4676(56.0 \%)$ & 3483 (55.9\%) & 1585 (55.9\%) & 667 (55.3\%) & 374 (57.3\%) & 191 (5I.2\%) \\
\hline University & $277 \mid(33.2 \%)$ & 2064 (33.1\%) & 907 (32.0\%) & 395 (32.7\%) & 215 (32.9\%) & 130 (34.9\%) \\
\hline \multicolumn{7}{|l|}{ Work status } \\
\hline Employed & 5505 (66.1\%) & 4048 (65.1\%) & 1809 (64.0\%) & 849 (70.6\%) & $422(64.8 \%)$ & 234 (63.1\%) \\
\hline Student & 95 (3.6\%) & $46(2.3 \%)$ & $34(3.7 \%)$ & II (3.3\%) & II (5.2\%) & II (8.8\%) \\
\hline Job seeker & 495 (19.0\%) & 380 (19.0\%) & 180 (19.4\%) & 85 (25.8\%) & 39 (18.6\%) & 29 (23.2\%) \\
\hline At home & $424(16.3 \%)$ & $308(15.4 \%)$ & 157 (16.9\%) & 51 (I5.5\%) & 32 (I5.2\%) & $16(12.8 \%)$ \\
\hline Invalid & $323(12.4 \%)$ & $270(13.5 \%)$ & $112(12.1 \%)$ & 57 (I7.3\%) & $42(20.0 \%)$ & 28 (22.4\%) \\
\hline Retired & 1272 (48.8\%) & 995 (49.8\%) & 445 (48.0\%) & $125(38.0 \%)$ & 86 (4I.0\%) & $4 \mathrm{I}(32.8 \%)$ \\
\hline
\end{tabular}

Notes: Data were missing for certain variables for some patients; percentages are calculated with respect to the total number of patients ( $\mathrm{N}$ ) for whom data were available. Since many patients presented comorbidities, these columns are not mutually exclusive.

Abbreviations: MDE, major depressive episode; GAD, generalised anxiety disorder; SAD, social anxiety disorder; PD, panic disorder; OCD, obsessive-compulsive disorder. 
was $-3.9,-4.2$, and -4.0 respectively; these changes were all significant as compared with baseline $(P<0.001)$. At this visit, the SDS subscores had decreased by at least one point for $90.0 \%$ (work/school), $92.8 \%$ (social life), and $91.1 \%$ (family life/home responsibilities) of patients. At the 6 -week study visit, the SDS dimension scores had improved by approximately 2.5 points (Table 3 ). Similar SDS scores at inclusion and at both the 6-week and 12-week follow-up visit were observed in all diagnostic subgroups (Table 3).

At inclusion, $77.1 \%$ of patients $(n=6449)$ scored $\geq 7$ (marked or extreme disability) on at least one dimension of the SDS and $29.4 \%(n=2467)$ scored $\geq 7$ on all three dimensions. At the 12-week follow-up, the proportion of patients scoring $\geq 7$ on any dimension had decreased to $9.2 \%(n=604)$ and the proportion scoring $\geq 7$ on all dimensions to $2.7 \%(\mathrm{n}=178)$. The proportion of patients scoring $<4$ (no or mild disability) on all three SDS dimensions rose over the course of the study from $2.4 \%(n=202)$ to $64.6 \%(n=4261)$. Functional remission rates defined as an SDS subscore of 0 were $18.0 \%$ for the work/school dimension, $16.8 \%$ for the social activities dimension, and $19.5 \%$ for the family life dimensions. Using a subscore of $\leq 2$ as the threshold, remission rates were $56.8 \%, 55.0 \%$, and $58.0 \%$, respectively. The distribution of SDS dimension scores over the course of the study is presented in Figure 2.

The number of days of activity (work/school) lost due to symptoms over the previous week decreased from $2.3 \pm 2.7$ days (median 1 ; interquartile range $0-5$ ) at inclusion to $0.5 \pm 1.5$ days (median 0 ; interquartile range $0-0$ ) at the 12-week follow-up visit (mean change $-1.8 \pm 2.7$ days; $P<0.001)$ and the number of days of impaired productivity decreased from $4.4 \pm 2.5$ days (median 5; interquartile

Table 3 Sheehan Disability Scale scores over the course of the study

\begin{tabular}{|c|c|c|c|c|c|c|}
\hline & $\begin{array}{l}\text { Total } \\
(N=8396)\end{array}$ & $\begin{array}{l}\text { MDE } \\
(N=6270)\end{array}$ & $\begin{array}{l}\text { GAD } \\
(N=2848)\end{array}$ & $\begin{array}{l}\text { SAD } \\
(N=12 \mid 3)\end{array}$ & $\begin{array}{l}P D \\
(N=656)\end{array}$ & $\begin{array}{l}\text { OCD } \\
(N=373)\end{array}$ \\
\hline \multicolumn{7}{|l|}{ Follow-up at $6 \pm 2$ weeks } \\
\hline Work/school activities & $N=5734$ & $N=4207$ & $N=1912$ & $N=831$ & $N=450$ & $N=275$ \\
\hline Score at inclusion & $6.5 \pm 2.2$ & $6.6 \pm 2.2$ & $6.4 \pm 2.2$ & $6.8 \pm 2.1$ & $6.7 \pm 2.3$ & $6.4 \pm 2.3$ \\
\hline Score at $6 \pm 2$ weeks & $4.1 \pm 2.3$ & $4.2 \pm 2.3$ & $4.1 \pm 2.3$ & $4.4 \pm 2.3$ & $4.2 \pm 2.4$ & $4.4 \pm 2.4$ \\
\hline Change from baseline & $-2.3 \pm 2.2$ & $-2.3 \pm 2.3$ & $-2.3 \pm 2.3$ & $-2.3 \pm 2.0$ & $-2.5 \pm 2.3$ & $-2.0 \pm 2.2$ \\
\hline Responders (\%) & $4672(81.5 \%)$ & $3406(81.0 \%)$ & 1537 (80.4\%) & $696(83.8 \%)$ & $369(82.0 \%)$ & $208(75.6 \%)$ \\
\hline Social activities & $N=6938$ & $N=5167$ & $N=2300$ & $N=958$ & $N=530$ & $N=305$ \\
\hline Score at inclusion & $6.8 \pm 1.9$ & $6.9 \pm 1.8$ & $6.6 \pm 2.0$ & $6.9 \pm 1.8$ & $7.1 \pm 1.9$ & $6.8 \pm 2.1$ \\
\hline Score at $6 \pm 2$ weeks & $4.2 \pm 2.2$ & $4.3 \pm 2.2$ & $4.1 \pm 2.2$ & $4.4 \pm 2.2$ & $4.3 \pm 2.3$ & $4.5 \pm 2.4$ \\
\hline Change from baseline & $-2.6 \pm 2.2$ & $-2.6 \pm 2.2$ & $-2.5 \pm 2.2$ & $-2.6 \pm 2.1$ & $-2.8 \pm 2.3$ & $-2.3 \pm 2.3$ \\
\hline Responders (\%) & $5882(84.8 \%)$ & $4382(84.8 \%)$ & $1904(82.8 \%)$ & $814(85.0 \%)$ & $459(86.6 \%)$ & $248(81.3 \%)$ \\
\hline Family life & $N=6936$ & $N=5165$ & $N=2299$ & $N=958$ & $N=529$ & $N=305$ \\
\hline Score at inclusion & $6.5 \pm 2.0$ & $6.7 \pm 1.9$ & $6.3 \pm 2.0$ & $6.3 \pm 2.1$ & $6.3 \pm 2.2$ & $6.6 \pm 2.2$ \\
\hline Score at $6 \pm 2$ weeks & $3.9 \pm 2.2$ & $4.1 \pm 2.2$ & $3.9 \pm 2.2$ & $3.9 \pm 2.2$ & $3.8 \pm 2.3$ & $4.2 \pm 2.3$ \\
\hline Change from baseline & $-2.5 \pm 2.3$ & $-2.6 \pm 2.3$ & $-2.4 \pm 2.3$ & $-2.3 \pm 2.2$ & $-2.5 \pm 2.4$ & $-2.4 \pm 2.2$ \\
\hline Responders (\%) & $5723(82.5 \%)$ & $4283(82.9 \%)$ & $1863(81.0 \%)$ & 779 (81.3\%) & $427(80.7 \%)$ & $25 \mathrm{I}(82.3 \%)$ \\
\hline \multicolumn{7}{|c|}{ Follow-up at $12 \pm 3$ weeks } \\
\hline Work/school activities & $N=5388$ & $N=3949$ & $N=1762$ & $N=791$ & $N=423$ & $N=263$ \\
\hline Score at inclusion & $6.5 \pm 2.2$ & $6.6 \pm 2.2$ & $6.4 \pm 2.2$ & $6.7 \pm 2.1$ & $6.7 \pm 2.2$ & $6.4 \pm 2.2$ \\
\hline Score at $12 \pm 3$ weeks & $2.6 \pm 2.2$ & $2.6 \pm 2.2$ & $2.6 \pm 2.2$ & $2.8 \pm 2.2$ & $2.5 \pm 2.1$ & $2.9 \pm 2.2$ \\
\hline Change from baseline & $-3.9 \pm 2.6$ & $-3.9 \pm 2.6$ & $-3.8 \pm 2.6$ & $-3.9 \pm 2.5$ & $-4.2 \pm 2.5$ & $-3.5 \pm 2.4$ \\
\hline Responders (\%) & $4850(90.0 \%)$ & $3548(89.8 \%)$ & 1572 (89.2\%) & 719 (90.9\%) & 391 (92.4\%) & 234 (89.0\%) \\
\hline Social activities & $N=6586$ & $N=4890$ & $N=215 \mid$ & $N=917$ & $N=502$ & $N=297$ \\
\hline Score at inclusion & $6.8 \pm 1.9$ & $6.9 \pm 1.8$ & $6.6 \pm 1.9$ & $6.9 \pm 1.8$ & $7.1 \pm 1.9$ & $6.8 \pm 2.0$ \\
\hline Score at $12 \pm 3$ weeks & $2.6 \pm 2.1$ & $2.7 \pm 2.2$ & $2.7 \pm 2.2$ & $2.7 \pm 2.1$ & $2.6 \pm 2.0$ & $3.1 \pm 2.2$ \\
\hline Change from baseline & $-4.2 \pm 2.5$ & $-4.2 \pm 2.5$ & $-4.0 \pm 2.5$ & $-4.2 \pm 2.4$ & $-4.5 \pm 2.3$ & $-3.8 \pm 2.5$ \\
\hline Responders (\%) & $6111(92.8 \%)$ & $4540(92.8 \%)$ & 1972 (91.7\%) & 862 (94.0\%) & $485(96.6 \%)$ & 269 (90.6\%) \\
\hline Family life & $N=6587$ & $N=4890$ & $N=2153$ & $N=917$ & $N=502$ & $N=296$ \\
\hline Score at inclusion & $6.5 \pm 2.0$ & $6.7 \pm 1.9$ & $6.3 \pm 2.1$ & $6.2 \pm 2.1$ & $6.3 \pm 2.2$ & $6.5 \pm 2.2$ \\
\hline Score at $12 \pm 3$ weeks & $2.4 \pm 2.1$ & $2.5 \pm 2.2$ & $2.5 \pm 2.1$ & $2.5 \pm 2.1$ & $2.3 \pm 2.0$ & $2.8 \pm 2.1$ \\
\hline Change from baseline & $-4.0 \pm 2.5$ & $-4.1 \pm 1.8$ & $-3.8 \pm 2.6$ & $-3.7 \pm 2.5$ & $-4.0 \pm 2.4$ & $-3.7 \pm 2.5$ \\
\hline Responders (\%) & 6003 (91.1\%) & 4470 (91.4\%) & 1972 (91.7\%) & 827 (90.2\%) & $466(92.8 \%)$ & 267 (90.2\%) \\
\hline
\end{tabular}

Note: Responders are defined as those patients whose SDS score decreases by at least one point.

Abbreviations: MDE, major depressive episode; GAD, generalised anxiety disorder; SAD, social anxiety disorder; PD, panic disorder; OCD, obsessive-compulsive disorder. 
A

Work/school dimension

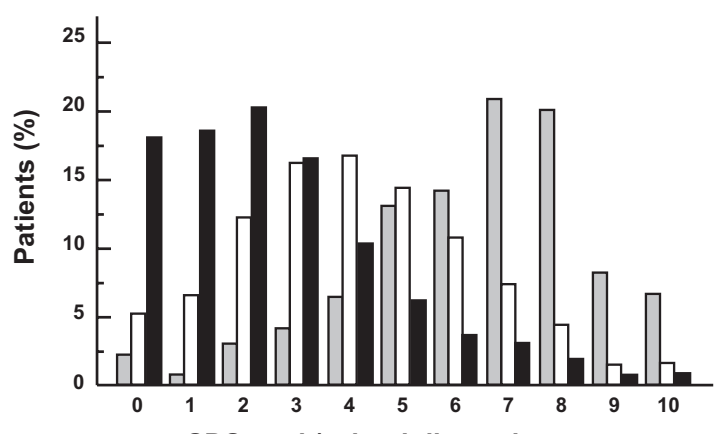

SDS work/school dimension score

B

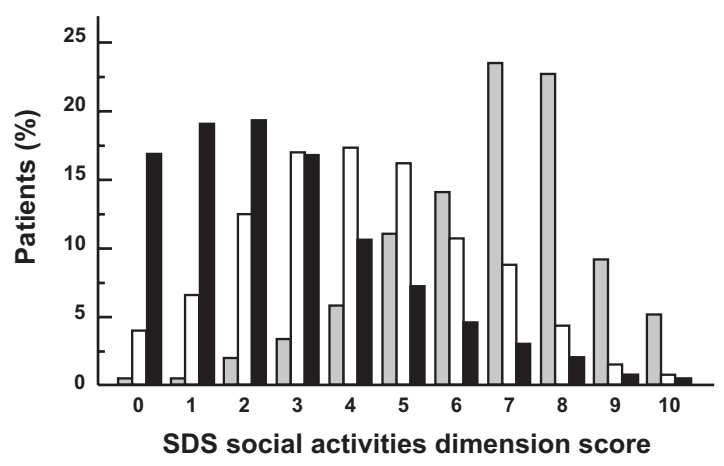

C

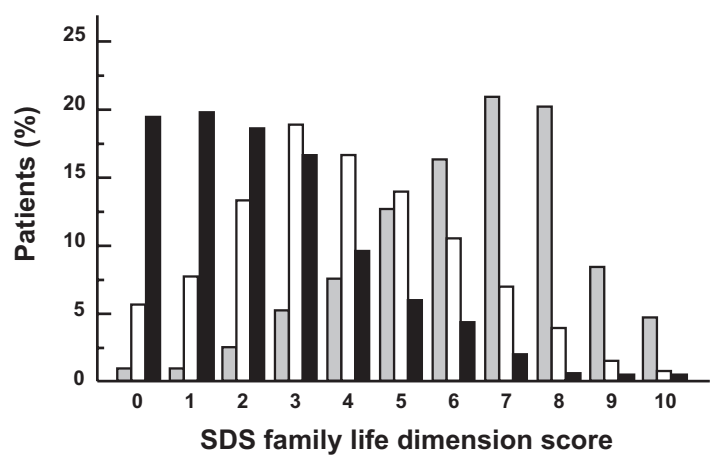

Figure 2 Distribution of Sheehan Disability Scale scores over the course of the study Notes: Grey represents inclusion visit; white represents 6-week follow-up visit; black represents I2-week follow-up visit.

range 2-7) to $0.9 \pm 1.8$ days (median 0 , interquartile range $0-1$; mean change $-3.5 \pm 2.8$ days; $P<0.001$ ). For the 6617 patients assessed at both inclusion and 12 weeks, the number of patients with no days lost in the previous week increased from $2861(43.2 \%)$ at inclusion to $5333(80.6 \%)$ at 12 weeks, and the number of patients with no days of impaired productivity increased from 851 (12.9\%) to 4193 (63.4\%).

Anxiety and mood symptoms as perceived by the patient were rated using the HADS. The mean anxiety dimension score decreased over the course of the study from $13.1 \pm 3.4$ points to $5.6 \pm 3.6$ points (mean change $-7.4 \pm 4.4$ points) and the mean depression dimension score from $13.5 \pm 4.0$ to $5.4 \pm 4.2$ points (mean change $-8.1 \pm 5.3$ points). In both cases, the change from baseline was statistically significant $(P<0.001)$. The proportion of patients scoring $\geq 8$, corresponding to clinically relevant symptoms, decreased from $93.0 \%$ at inclusion to $25.8 \%$ at study end for the anxiety dimension and from $91.8 \%$ to $29.7 \%$ for the depression dimension.

Severity of mental illness was rated by the investigators using the CGI-S. At inclusion, the mean CGI-S score was $4.6 \pm 0.8$ (median 5, corresponding to "markedly ill"). At the 12 -week study visit, the score had decreased to $2.6 \pm 1.3$ (median 2, corresponding to "borderline mentally ill"), a mean improvement of $2.0 \pm 1.4$ points. The distribution of CGI-S scores is presented in Figure 3. This distribution was significantly different between inclusion and the 12-week follow-up ( $P<0.001$; Chi-square test). The mean CGI-I score at the 12-week follow-up was $2.0 \pm 0.9$ (median 2, corresponding to "markedly improved") and 4928 patients (74.5\%) were responders (CGI-I $=1$ or 2 ) and 3433 patients $(51.9 \%)$ were in remission (CGI-S $=1$ or 2$)$.

\section{Self-reported disability versus physician-rated symptoms}

The relationship between self-reported disability at inclusion (rated with the SDS) and physician-rated severity of clinical symptoms (rated with the CGI-S) was evaluated. Over the low disease severity range from CGI-S scores 1 to 4 , corresponding to normal to moderately ill, the SDS scores did not vary with CGI-S score and were close to 5 for all severity grades. Nevertheless, it should be noted that the number of patients with CGI-S scores of 1 or 2 was low ( $n=212 ;<3 \%$ of all patients). However, between CGI-S scores 3 (mildly ill) and 7 (among the most extremely ill patients), mean SDS scores rose from around 5 to $>8$ (Figure 4). At each CGI-S grade, scores on all three SDS dimensions were similar. The proportion of patients reporting severe disability (SDS score $\geq 7$ ) rose from $26 \%$ to $31 \%$ (according to the dimension) at CGI-S $=3$ (mildly ill) to $84 \%-90 \%$ at CGI-S $=6$ (severely ill).

With respect to physician-rated improvement (CGI-I), the mean change in SDS dimension scores was correlated with the CGI-I score (Figure 4). Again, all three SDS dimension scores varied in a coherent fashion. The proportion of patients with all three SDS dimension scores improved by at least one point increased from $28.0 \%$ at CGI-I $=6$ (much worse) and $31.0 \%$ at CGI-I $=4$ (no change) to $93.0 \%$ at CGI-I $=1$ 


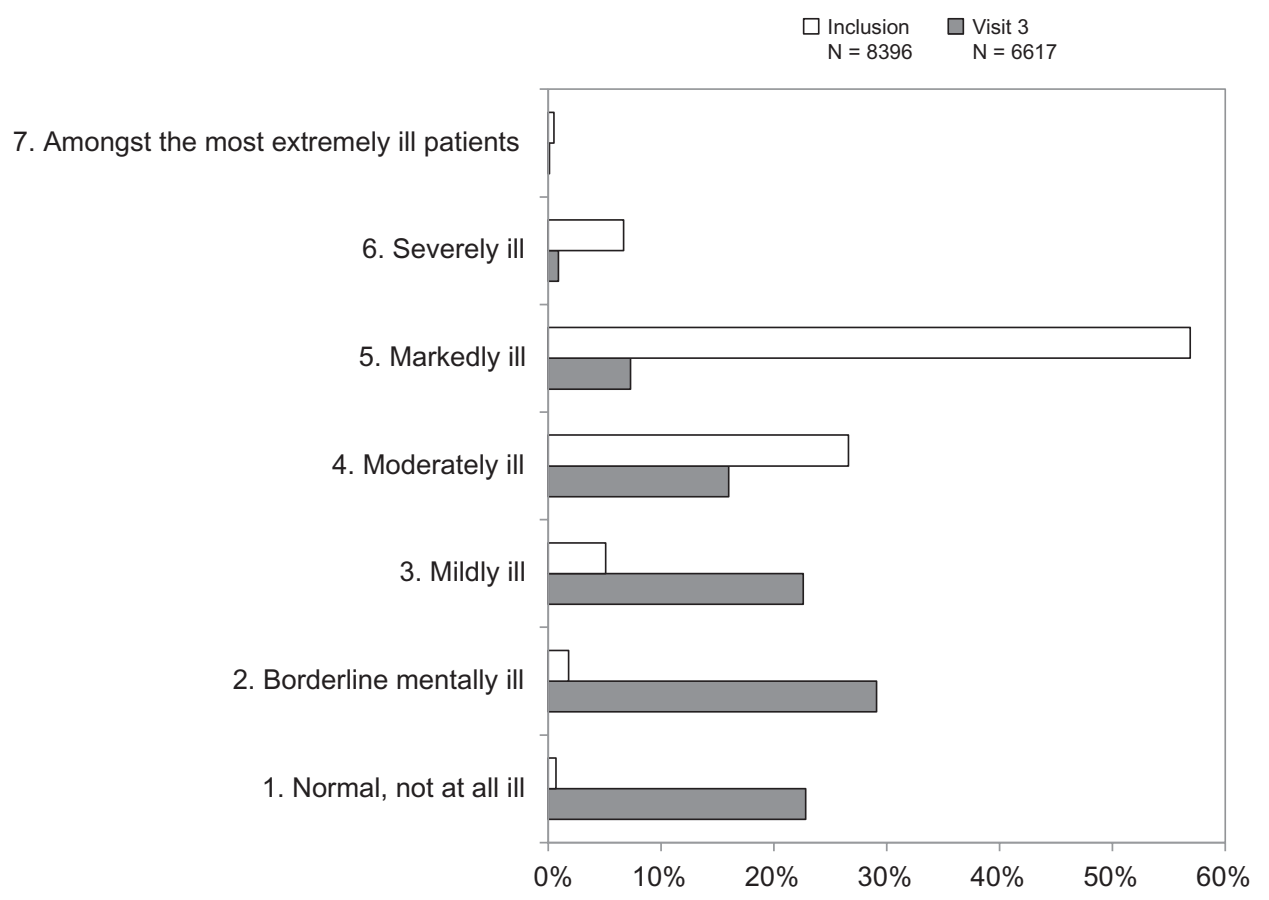

Figure 3 Clinical Global Impression-Severity score at inclusion (open columns) and the I2-week follow-up visit (filled columns).

Note: The analysis was performed on patients for whom data were available at both visits.

(very much improved). The Spearman coefficients for the correlation between CGI-I improvement score and the change in SDS score between baseline and study end were 0.51 for the work dimension, 0.54 for the social dimension, and 0.50 for the family dimension.

\section{Discussion}

The principal findings of the DéPasS study were the high proportion of patients with mood or anxiety disorders in primary care who reported severe disability and who reported a significant improvement of disability following initiation of an antidepressant treatment.

For patients with a major depressive episode, the results from this study are consistent with the WMH surveys, which reported that $65.8 \%$ of individuals with major depressive disorder in high-income countries reported severe disability, ${ }^{8}$ and with clinical trials of patients suffering from a major depressive episode, who presented SDS dimension scores
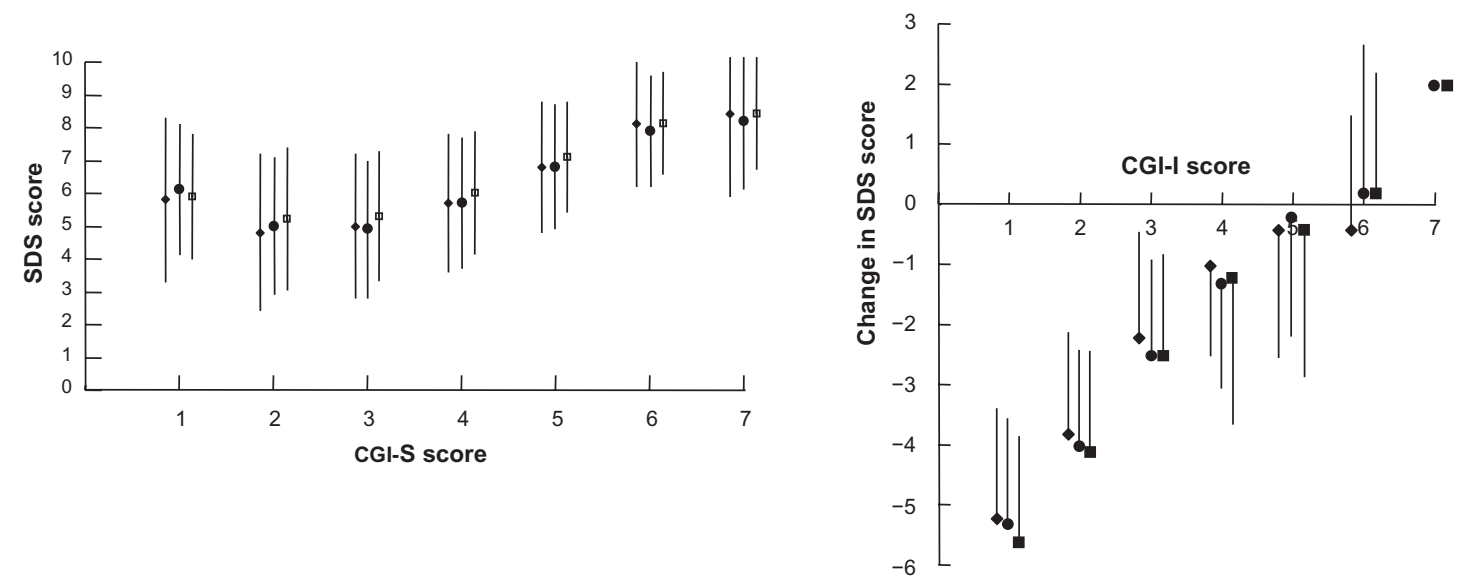

Figure 4 Left shows correlation between CGI-C score and SDS dimension scores at inclusion.

Notes: Right shows correlation between CGI-I score and change in SDS dimension scores between inclusion and I2-week follow-up. The three SDS dimensions are indicated separately: Work-school dimension; social activity dimension; and • family life dimension. Data are presented as the mean SDS score with the standard deviation (in the right panel, these are presented unilaterally only for clarity).

Abbreviations: CGI-S, Clinical Global Impression-Severity; SDS, Sheehan Disability Scale; CGI-I, Clinical Global Impression-Improvement. 
at baseline ranging from 6.0 (work/school dimension) to 6.8 (social activities dimension). ${ }^{13}$ Although previous data would suggest that patients with anxiety disorders report less disability than those with major depressive disorder, we did not find major differences in SDS dimension scores between the various diagnostic groups included in the study. ${ }^{1}$ This may be explained by extensive comorbidity of depression with anxiety disorders in our patients, in contrast with those included in clinical trials, where investigators are careful to exclude patients with comorbidities.

After initiation of antidepressant treatment in our patient cohort, the proportion of patients reporting severe disability on any dimension in the previous week declined from $66.3 \%$ to $7.0 \%$, a mean decrease in SDS score of 3.9-4.2 (according to the dimension), a response rate of $90.0 \%-92.8 \%$, and a remission rate of $17.1 \%-19.5 \%$. These treatment effects are generally consistent with those reported in randomized clinical trials of selective serotonin reuptake inhibitors and serotonin/noradrenaline reuptake inhibitors in anxiety disorders or in major depressive disorder. ${ }^{13}$ Two large observational studies have evaluated self-reported disability with the SDS, both of which included over 5000 patients with major depressive disorder starting treatment with selective serotonin reuptake inhibitors. ${ }^{17,18}$ The first, conducted in the US, reported a baseline SDS score of 6.0-6.8 (depending on the dimension), a decrease in score 8 weeks after starting treatment of 2.5-2.9 points, and a proportion of patients with mild or no disability (score $<4$ ) of $51 \%-58 \% .{ }^{17}$ The second study, performed in Greece, reported a proportion of patients with mild or no disability after 12 weeks of treatment of $80 \%-84 \%{ }^{18}$ The results of the DéPasS study are consistent with these previous findings. Nonetheless, it should be noted that, for the DéPasS study, as for other noncomparative observational studies, a causal relationship between antidepressant treatment and reduction in disability cannot be demonstrated formally in the absence of an untreated control group.

Reductions in disability were already observed at the 6-week follow-up visit. It is possible that further improvement would have been seen if follow-up was continued up to 24 weeks, as recommended in current practice guidelines. ${ }^{19,20}$ Moreover, the mean number of days "out of role" due to symptoms and the mean number of days with reduced productivity in the previous week were reduced by a factor of four after 12 weeks of treatment, to less than one day per week. These findings are again consistent with the previous Greek study. ${ }^{18}$ The extent of the decrease in SDS score was correlated with the reduction in psychiatric symptoms, as measured by the CGI-I, as reported in previous studies. ${ }^{21,22}$
In parallel, the proportion of patients with clinically significant symptoms (HADS score $\geq 7$ ) decreased to $<30 \%$. These observations suggest that the SDS is both a relevant and valid measure of disease-related disability in this patient population.

The finding that self-reported disability is improved to a significant extent after starting antidepressant treatment, together with the observation that around two-thirds of patients in the study were severely disabled, suggests that such treatment is both justified and useful, and that clinical practice observed in a primary care context in this study is appropriate and effective.

At inclusion, we noted that even patients rated by their physicians as mildly ill report significant disability. The observation of significant disability in patients with mild depression or anxiety disorders has been made previously. ${ }^{23}$ More surprising was the finding of an apparent "floor effect", with patients rated between 1 (not ill) and 4 (moderately ill) on the CGI-S having essentially similar levels of self-reported disability. This finding needs to be interpreted with caution, firstly because of the low patient numbers at the bottom end of the CGI-S scale and, secondly, because it is unclear why a physician who diagnosed a patient with a mood or anxiety disorder sufficiently severe to warrant treatment would rate the patient at 1 on the CGI-S (normal, not ill at all). This may suggest that the CGI-S was not understood or used appropriately in some cases.

Given the naturalistic setting of the study, participating general practitioners made a diagnosis on the basis of their own experience and judgment, rather than using a standardized diagnostic algorithm, and no attempt was made to ascertain whether the diagnoses given actually corresponded to DSM-IV Text Revision criteria. However, a survey of primary care physicians prescribing selective serotonin reuptake inhibitors in France revealed that operational diagnoses of general practitioners were generally consistent with a DSM-IV Text Revision diagnosis made a posteriori. ${ }^{24}$

The DéPasS study has several strengths and limitations. Its strengths include the large number of participating general practitioners and patients $(>8000)$ and the naturalistic treatment setting. The investigator sample corresponds to approximately $3 \%$ of all general practitioners in France. Nonetheless, because physicians who declined to participate may see different types of patients or have different treatment practices than participants, these results may not be representative of all general practitioners. Although the proportion of patients who failed to return for a follow-up visit was relatively low $(-20 \%)$, it cannot be excluded that 
treatment outcome differed between the patients analyzed and those who failed to return for a follow-up visit.

In conclusion, this large naturalistic study in primary care in France showed that patients presenting to general practitioners for mood and anxiety disorders report significant disability and that this can be reduced effectively and rapidly after antidepressant treatment is initiated. Relief of self-reported disability is correlated with clinical improvement.

\section{Disclosure}

AG, FL, and BM were members of the steering committee of the study and received honoraria for their participation. AC and SG are employees of Lundbeck SAS, the manufacturer of escitalopram.

\section{References}

1. Kessler RC, Aguilar-Gaxiola S, Alonso J, et al. The global burden of mental disorders: an update from the WHO World Mental Health (WMH) surveys. Epidemiol Psichiatr Soc. 2009;18(1):23-33. Italian.

2. Lepine JP, Gasquet I, Kovess V, et al. Prevalence and comorbidity of psychiatric disorders in the French general population. L'Encephale. 2005;31(2):182-194. French.

3. Alonso J, Angermeyer MC, Bernert S, et al. 12-Month comorbidity patterns and associated factors in Europe: results from the European Study of the Epidemiology of Mental Disorders (ESEMeD) project. Acta Psychiatr Scand. 2004;420:28-37.

4. Kessler RC, Berglund P, Demler O, et al. The epidemiology of major depressive disorder: results from the National Comorbidity Survey Replication (NCS-R). JAMA. 2003;289(23):3095-3105.

5. Henderson S, Andrews G, Hall W. Australia's mental health: an overview of the general population survey. Aust $N Z$ J Psychiatry. 2000;34(2):197-205.

6. Regier DA, Narrow WE, Kuhl EA, Kupfer DJ. The conceptual development of DSM-V. Am J Psychiatry. 2009;166(6):645-650.

7. World Health Organization. The Global Burden of Disease: 2004 Update. Geneva, Switzerland: World Health Organization; 2004.

8. Ormel J, Petukhova M, Chatterji S, et al. Disability and treatment of specific mental and physical disorders across the world. Br J Psychiatry. 2008;192(5):368-375.

9. Esposito E, Wang JL, Williams JV, Patten SB. Mood and anxiety disorders, the association with presenteeism in employed members of a general population sample. Epidemiol Psichiatr Soc. 2007;16(3): 231-237. Italian.
10. Lepine JP. Epidemiology, burden, and disability in depression and anxiety. J Clin Psychiatry. 2001;62 Suppl 13:4-10.

11. Alonso J, Angermeyer MC, Bernert S, et al. Disability and quality of life impact of mental disorders in Europe: results from the European Study of the Epidemiology of Mental Disorders (ESEMeD) project. Acta Psychiatr Scand Suppl. 2004;420:38-46.

12. Papakostas GI, Petersen T, Mahal Y, Mischoulon D, Nierenberg AA, Fava M. Quality of life assessments in major depressive disorder: a review of the literature. Gen Hosp Psychiatry. 2004;26(1):13-17.

13. Sheehan KH, Sheehan DV. Assessing treatment effects in clinical trials with the discan metric of the Sheehan Disability Scale. Int Clin Psychopharmacol. 2008;23(2):70-83.

14. National Institute of Mental Health. CGI, Clinical Global Impression. In: Guy W, editor. ECDEU Assessment Manual of Psychopharmacology Review. Rockville, MD: National Institute of Mental Health; 1976.

15. Sheehan DV, Harnett-Sheehan K, Raj BA. The measurement of disability. Int Clin Psychopharmacol. 1996;11 Suppl 3:89-95.

16. Zigmond AS, Snaith RP. The Hospital Anxiety and Depression Scale. Acta Psychiatr Scand. 1983;67(6):361-370.

17. Rush AJ, Bose A. Escitalopram in clinical practice: results of an open-label trial in a naturalistic setting. Depress Anxiety. 2005; 21(1):26-32.

18. Stamouli SS, Yfantis A, Lamboussis E, et al. Escitalopram in clinical practice in Greece: treatment response and tolerability in depressed patients. Expert Opin Pharmacother. 2009;10(6):937-945.

19. Bandelow B, Zohar J, Hollander E, et al. World Federation of Societies of Biological Psychiatry (WFSBP) guidelines for the pharmacological treatment of anxiety, obsessive-compulsive and post-traumatic stress disorders - first revision. World J Biol Psychiatry. 2008;9(4): 248-312.

20. Bauer M, Bschor T, Pfennig A, et al. World Federation of Societies of Biological Psychiatry (WFSBP) guidelines for biological treatment of unipolar depressive disorders in primary care. World J Biol Psychiatry. 2007;8(2):67-104.

21. Stein DJ, Bandelow B, Dolberg OT, Andersen HF, Baldwin DS. Anxiety symptom severity and functional recovery or relapse. Ann Clin Psychiatry. 2009;21(2):81-88.

22. Bandelow B, Baldwin DS, Dolberg OT, Andersen HF, Stein DJ. What is the threshold for symptomatic response and remission for major depressive disorder, panic disorder, social anxiety disorder, and generalized anxiety disorder? J Clin Psychiatry. 2006;67(9):1428-1434.

23. Lepine JP, Gastpar M, Mendlewicz J, Tylee A. Depression in the community: the first pan-European study DEPRES (Depression Research in European Society). Int Clin Psychopharmacol. 1997;12(1):19-29.

24. Martinez L, Gasquet I, Lenne X, et al. SSRI antidepressant prescription: compliance with recommendations? Rev Prat Med Gen. 2003; 17(623):1093-1098. French.
Neuropsychiatric Disease and Treatment

\section{Publish your work in this journal}

Neuropsychiatric Disease and Treatment is an international, peerreviewed journal of clinical therapeutics and pharmacology focusing on concise rapid reporting of clinical or pre-clinical studies on a range of neuropsychiatric and neurological disorders. This journal is indexed on PubMed Central, the 'PsycINFO' database and CAS.

\section{Dovepress}

The manuscript management system is completely online and includes a very quick and fair peer-review system, which is all easy to use. Visit http://www.dovepress.com/testimonials.php to read real quotes from published authors. 